\title{
Eje psicosocial familiar: reporte del diseño e implementación de un programa docente para residentes de los programas de postítulo en Medicina Familiar
}

\author{
Pamela Rojas ${ }^{{ }^{*}}$, Gabriela Soto ${ }^{2}$, Philippa Moore ${ }^{1}$, Paulina Rojas ${ }^{1}$, Javiera Léniz ${ }^{1}$, Gladys Moreno ${ }^{1}$
}

Resumen - Cada vez más se acepta que la atención integral en salud requiere un foco de abordaje que esté puesto en la persona y su familia y no solo en la enfermedad o sus consecuencias. En concordancia con esta idea, con los objetivos propuestos para los Programas de Postítulo de Medicina Familiar de la Pontificia Universidad Católica de Chile, con las necesidades percibidas por docentes y residentes y con la literatura disponible en relación con el currículo de esta especialidad, se trabajó en la revisión de la formación que los residentes de estos programas recibían con respecto a aspectos psicosociales y familiares.

En respuesta a este análisis, se trabajó en la definición de las competencias específicas en el ámbito psicosocial familiar deseables para los egresados de nuestros Programas de Postítulo. De acuerdo con las conclusiones de este trabajo, se revisó y replanteó el currículo vigente.

Este artículo resume y presenta el proceso de diseño e implementación de un programa docente específico para la formación en competencias del ámbito psicosocial familiar para los residentes de los programas mencionados.

Palabras Clave: Medicina Familiar, Atención integral, Salud, Psicosocial.

\begin{abstract}
Increasingly it is accepted that comprehensive health care approach requires that the focus has to be placed on the person and his family and not just the disease or its consequences. Consistent with this idea, the proposed objectives of the post graduate programs of Family Medicine at Pontifical Catholic University of Chile, focused on the perceived needs of teachers and residents based on the literature available on this area, we worked in reviewing the training that residents of these programs received regarding family and psychosocial aspects.
\end{abstract}

In response to this analysis, we worked on the definition of specific skills in the family psychosocial environment desirable for graduates from our post graduate programs. According to the findings of this work the current curriculum was reviewed and reconsidered.

This article summarizes and presents the process of designing and implementing a specific educational program for training in skills of family psychosocial environment for the residents of these programs.

Palabras Clave: General Practicioner, Integral assistance, Health, Psychosocial.

Fecha de envío: 20 de Mayo de 2012 - Fecha de aceptación: 21 de Septiembre de 2012

\section{Introducción:}

La comprensión global de una persona exige mirar más allá de ella y observar su entorno. Esto, traducido a la salud, implica una comprensión de los síntomas y problemas de salud en el contexto de las personas y sus otros significados; y la necesidad de actuar en concordancia con estos hallazgos (McDaniel et al., 2005). De este enfoque centrado en la persona, se deriva la necesidad de abordar el trabajo en salud desde una perspectiva biopsicosocial.

El abordaje biopsicosocial en salud se sustenta en la interacción que se ha documentado entre quién consulta y su entorno, donde la familia juega un rol central. Esta interacción incluye el rol condicionante que ella cumple en la evolución de los

1) Departamento Medicina Familiar Pontificia Universidad Católica de Chile.

2) Unidad Medicina Familiar Centro Médico San Joaquín Red Salud UC. Pontificia Universidad Católica de Chile.

*Autor de correspondencia: projasgo@uc.cl 
problemas de salud; en la transmisión de hábitos, creencias, representaciones sociales y prácticas sanitarias; en el modelaje que ella realiza en términos de la resolución de problemas; en el impacto que tiene el estrés familiar propio de las transiciones del ciclo vital sobre la salud; en la función adaptativa de algunos síntomas somáticos; y en el rol de unidad prestadora de cuidado que cumple la familia frente al paciente (Hidalgo \& Carrasco, 2002; McDaniel et al., 2005; Colapinto, 2006).

La efectividad del enfoque centrado en la persona y la familia en salud ha sido documentada por Stewart, McWhinney y otros (Stewart et al., 1999; Stewart et al., 2000). La evidencia da cuenta del rol que cumple la familia en el desarrollo y evolución de problemas de salud tan diversos como el asma infantil (Chen et al., 2007), los problemas de conducta de la infancia (Kim-Cohen et al., 2005) y la patología cardiovascular del adulto (Hartmann et al., 2010).

Los Programas de Postítulo en Medicina Familiar de la Facultad de Medicina de la Pontificia Universidad Católica de Chile se crearon el año 1993 buscando fortalecer la atención primaria en salud. Para lograr su objetivo de formar "médicos especialistas en los problemas prevalentes vinculados a la atención médica ambulatoria, (...) con un enfoque de salud familiar", el residente en medicina familiar adquirir, entre otras, un conjunto de competencias específicas orientadas al área psicosocial y familiar. En este contexto, surge la necesidad de evaluar la docencia en los aspectos psicosociales y familiares que contemplaban los currículos de los Programas de Formación en Medicina Familiar de la Pontificia Universidad Católica de Chile, y eventualmente replantear la malla curricular en relación con estos aspectos.

\section{Descripción de la experiencia:}

Para el desarrollo de este trabajo se usó como base el modelo de desarrollo curricular de Kern (2010). La metodología utilizada incluyó 6 etapas:

\section{Identificación del problema:}

Durante el año 2008 se diseñó una encuesta escrita de evaluación de los Programas de Medicina Familiar. La encuesta consistía en 68 preguntas que permitía a los residentes y docentes evaluar cuantitativa y cualitativamente sus objetivos y actividades. La encuesta fue completada por el $100 \%$ de los residentes y el $70 \%$ de los docentes. De ella se rescató que uno de los aspectos por mejorar en ambos Programas era la docencia en las áreas psicosocial y familiar (Pontificia Universidad Católica de Chile; Escuela de Medicina; Departamento de Medicina Familiar; Programa de Postítulo de Medicina Familiar, 2008b).

\section{Evaluación de las necesidades:}

Para profundizar en la perspectiva de los residentes se realizaron 4 grupos focales; uno con residentes de primer año, otro con residentes de segundo año, y uno con residentes de tercer año. El último grupo estuvo integrado por ex residentes de los Programas, egresados los últimos 2 años, con el objetivo de profundizar en las necesidades de formación percibidas por estos. Los grupos focales fueron realizados entre noviembre y diciembre de 2008, y analizados por un psicólogo externo a los programas (Pontificia Universidad Católica de Chile; Escuela de Medicina; Departamento de Medicina Familiar; Programa de Postítulo de Medicina Familiar, 2008a).

Adicionalmente, se realizó una revisión no sistemática de la literatura con el fin de identificar necesidades de formación en el área psicosocial y familiar para el Médico de Familia. Esta revisión incluyó un Perfil de Competencias Indispensables para el Médico de Atención Primaria Chileno (Montero et al., 2009)2009, el Perfil de Competencias Generales para Médicos Canadienses (Frank, 2005), el Perfil de Competencias para Médicos Familiares Canadienses (Tannenbaum et al., 2009), y el Plan de Desarrollo Estratégico del Departamento de Medicina Familiar 2008-2013 (Pontificia Universidad Católica de Chile; Escuela de Medicina; Departamento de Medicina Familiar, 2009).

\section{Definición de competencias:}

En marzo del 2009 se realizaron sesiones grupales de discusión participativa no estructurada, en las que se consensuaron y definieron las competencias del área psicosocial familiar que debían adquirir los residentes en cada uno de sus 3 años de formación. Las sesiones fueron conducidas por el equipo de posgrado, e incluyeron 3 médicos familiares mención niños, 4 médicos familiares mención adultos, 1 psiquiatra, y 2 psicólogas clínicas. Todos los integrantes del equipo participante cumplían actividades docentes y asistenciales en la Pontificia Universidad Católica de Chile y su Red de Salud.

\section{Metodologías:}

El resultado de la evaluación de necesidades y la definición de competencias específicas en el ámbito psicosocial y familiar, dieron cuenta de la existencia de diversas instancias de formación en esta área en los currículos existentes (cursos, supervisión, trabajo con familias en los centros de salud, etc.). Se reveló, sin embargo, que estas actividades estaban diseñadas a partir de objetivos aislados, y que no se desprendían necesariamente de las competencias que debía adquirir un residente de Medicina 
Familiar. Por otro lado, las actividades se concentraban durante el primer año de formación, y su integración a la práctica clínica no estaba sistematizada. El resultado era la dificultad por parte de los residentes para contextualizar los contenidos tratados y vincularlos con su trabajo clínico.

A partir de las conclusiones del trabajo realizado y de las competencias consensuadas, el equipo de trabajo diseñó un programa académico denominado "Eje Psicosocial Familiar". El programa tiene como objetivo estructurar a través de los tres años de formación del residente un eje continuo y progresivo de actividades docente-asistenciales que les permita adquirir los conocimientos, habilidades y actitudes necesarias para su desempeño clínico en esta área.

A través de este programa se espera contribuir a la formación integral de los residentes de Medicina Familiar, facilitando la adquisición de competencias que les permitan la comprensión e intervención familiar, en el contexto de la salud.

El "Eje Psicosocial Familiar" se estructuró a partir de módulos, cada uno de ellos con objetivos específicos, métodos, estrategias de aprendizaje, y estructura de evaluación independiente. Los módulos incluyen actividades teóricas y prácticas relacionadas al cumplimiento de sus objetivos particulares, y se articulan entre sí.

A la metodología descrita, se suma un acompañamiento docente formal y sistemático, que busca que el residente tome conciencia de los procesos que desarrolla durante su exposición a nuevas experiencias, y actuar como guía para que pueda significarlos de manera constructiva.

El diseño del "Eje Psicosocial Familiar" busca facilitar un aprendizaje positivo y en espiral, de modo que cada contenido o habilidad que se adquiere da un nuevo sentido a lo que se trató con anterioridad. Tiene como meta que los residentes incrementen la aplicación de las competencias adquiridas, tanto en las dimensiones cognitiva, actitudinal y de habilidades, a sus atenciones clínicas habituales.

No existe otro programa similar en el cual se haya basado la formulación de esta modalidad.

\section{Plan e implementación:}

La implementación de este nuevo programa se inició en julio de 2010 para 9 residentes, pertenecientes al primer año de los Programas de Postítulo de Medicina Familiar de ese año. Estuvo a cargo del equipo docente, y fue liderado por un coordinador del mismo equipo.

El programa se encuentra funcionando actualmente con 2 cohortes de residentes, ingresados el 2010 y el 2011, respectivamente. Para mayo de 2012 se espera el ingreso de la tercera cohorte de residentes, grupo con el cual el programa completará la ejecución del ciclo de 3 años.

\section{Evaluación:}

Tiene como meta cubrir la mayor parte de las competencias consensuadas, haciéndolo de la manera más efectiva y eficiente posible. Su diseño y planificación supone abordar en paralelo evaluaciones de corte formativo y sumativo; tanto individuales como del programa; y dirigidas a medir el logro de objetivos medios y terminales (Mann, 2002; Holmboe, 2004; Kern, 2010).

Cada módulo diseñado para el "Eje Psicosocial Familiar" cuenta con un mecanismo de evaluación centrado en los aspectos cognitivos, actitudinales y de habilidades específicas. La evaluación es esencialmente formativa e incluye también una instancia de evaluación sumativa. Para ello se utilizan diversos instrumentos: pruebas escritas, informes de análisis de caso, observación directa de atenciones clínicas, filmación de atenciones y análisis con pautas estructuradas, etc. Está pendiente la evaluación de las competencias terminales del programa y su impacto en la práctica clínica de los residentes.

Con respecto a la evaluación del programa, se han realizado algunas evaluaciones preliminares, por parte de los residentes y docentes, sin embargo, esta área debe ser sistematizada. Esto, con el fin de evaluar la efectividad y sustentabilidad del programa, y eventualmente replantearse su estructura, contenido, diseño, metodología, e incluso objetivos, si la situación lo amerita (DaRosa et al., 2011).

\section{Proyecciones de desafíos}

El programa docente que se presenta enfrenta hoy 3 grandes desafíos: la evaluación, la integración docente asistencial y la investigación. En relación al primer punto, el programa propuesto debe avanzar hacia la evaluación de competencias terminales, para lo cual el desafío es la generación de espacios de evaluación colaborativos e integradores; y la identificación y desarrollo de instrumentos efectivos, eficientes, y aplicables a un contexto académico.

En relación con la integración docente asistencial, el desafío 
incluye la integración de la docencia del programa con el trabajo clínico regular de los equipos de salud de los Centros de Salud Familiar formadores. Esto, con el objeto no sólo de rescatar su experiencia y dar sustentabilidad al programa, sino además de estimular un trabajo en los centros bajo un perfil universitario, que promueva la reflexión, sistematización de experiencias, y la investigación.

En relación con la investigación, el Eje Psicosocial Familiar abre un nuevo espacio tanto en el contexto de la investigación clínica como en relación a metodologías docentes e instrumentos de evaluación. Al respecto, y a partir de este programa, se trabaja actualmente en dos proyectos financiados. El primero, cuyo objetivo es revisar el programa de formación del pregrado de Medicina de la Pontificia Universidad Católica de Chile, en relación con su caracterización y oportunidad de adquisición formal de contenidos, habilidades y actitudes en el área psicosocial familiar (Rojas et al., 2011). El segundo, orientado a sistematizar y pilotear la factibilidad de implementación de un modelo de atención familiar breve en atención primaria de salud pública y privada (Léniz et al., 2011).

\section{Discusión:}

La práctica de la Medicina Familiar exige la adquisición de competencias de diversa índole para brindar una óptima atención de salud. Los objetivos de los Programas de Postítulo en Medicina Familiar de la Pontificia Universidad Católica de Chile acogen este postulado; sin embargo, y de acuerdo al diagnóstico realizado era necesario fortalecer su aplicación práctica.

El trabajo de revisión y replanteamiento curricular, estructurado bajo el modelo de Kern y colaboradores, permitió identificar un problema y elaborar desde su diagnóstico una estrategia planificada y menos reactiva. Este proceso permitió a los residentes pasar desde un aprendizaje basado en componentes aislados a uno diseñado en función de las competencias que se espera que adquieran en el área psicosocial familiar; donde el foco es la persona humana y su contexto.

Los Programas de Postítulo de Medicina Familiar de la Pontificia Universidad Católica de Chile cuentan en la actualidad con un programa académico específico para el desarrollo del área psicosocial familiar. Su diseño permite integrar contenidos teóricos y prácticos, vinculando al residente a un escenario más real. Cuenta además con un espacio de acompañamiento docente sistemático, que permite al residente tomar conciencia y resignificar los procesos que desarrolla durante su formación. Con la incorporación de este programa académico a los currículos actuales de ambos Programas de Postítulo se espera contribuir a la formación integral de los residentes de Medicina Familiar, y promover un trabajo en salud más efectivo.

\section{Referencias:}

Colapinto J. (2006). Enfoque estructural. In Terapia familiar y de pareja, ed. Roizblatt A, pp. 209-225. Mediterráneo, Santiago, Chile.

Chen E, Chim LS, Strunk RC \& Miller GE. (2007). The role of the social environment in children and adolescents with asthma. American journal of respiratory and critical care medicine 176, 644-649.

DaRosa DA, Skeff K, Friedland JA, Coburn M, Cox S, Pollart S, O'Connell M \& Smith S. (2011). Barriers to effective teaching. Academic medicine: journal of the Association of American Medical Colleges $\mathbf{8 6}, \mathbf{4 5 3 - 4 5 9}$.

Frank J. (2005). The CanMEDS 2005 physician competency framework. Better standards. Better physicians. Better care. The Royal College of Physicians and Surgeons of Canada, Ottawa.

Hartmann M, Bazner E, Wild B, Eisler I \& Herzog W. (2010). Effects of interventions involving the family in the treatment of adult patients with chronic physical diseases: a meta-analysis. Psychotherapy and psychosomatics 79, 136-148.

Hidalgo C \& Carrasco E. (2002). Teorías acerca del funcionamiento familiar. In Salud familiar: un modelo de atención integral en la atención primaria, 2nd edn, ed. Hidalgo C \& Carrasco E, pp. 27-69. Pontificia Universidad Católica de Chile, Santiago, Chile.

Holmboe ES. (2004). Faculty and the observation of trainees' clinical skills: problems and opportunities. Academic medicine : journal of the Association of American Medical Colleges 79, 16-22.

Kern DE. (2010). Overview: a six-step approach to curriculum development. In Curriculum development for medical education: a six-step approach, 2nd edn, ed. Kern DE, Thomas PA \& Hughes MT, pp. 5-9. John's Hopkins University Press, Baltimore, Maryland.

Kim-Cohen J, Moffitt TE, Taylor A, Pawlby SJ \& Caspi A. (2005). Maternal depression and children's antisocial behavior: nature and nurture effects. Archives of general psychiatry 62, 173-181.

Léniz J, Moore P, Sotos G \& Rojas P. (2011). Protocolo: Sistematización de una intervención familiar breve en atención primaria. Fuente Financiamiento: Proyecto Intradepartamental Departamento Medicina Familiar. Pontificia Universidad Católica de Chile. 
Mann KV. (2002). Thinking about learning: implications for principle-based professional education. The Journal of continuing education in the health professions 22, 69-76.

McDaniel SH, Campbell TL, Hepworth J \& Lorenz A. (2005). Family-Oriented Primary Care. Springer-Verlag New York.

Montero J, Rojas MP, Castel J, Muñoz M, Brunner A, Sáez Y \& Scharager J. (2009). Competencias médicas requeridas para el buen desempeño en Centros de Salud Familiares en Chile. Revista médica de Chile 137, 1561-1568.

Pontificia Universidad Católica de Chile; Escuela de Medicina; Departamento de Medicina Familiar. (2009). Plan de desarrollo estratégico del Departamento de Medicina Familiar 2008-2013.

Pontificia Universidad Católica de Chile; Escuela de Medicina; Departamento de Medicina Familiar; Programa de Postítulo de Medicina Familiar. (2008a). Estudio cualitativo de atributos y satisfacción percibida del Programa de Postítulo de Medicina Familiar de la Pontificia Universidad Católica de Chile.

Pontificia Universidad Católica de Chile; Escuela de Medicina; Departamento de Medicina Familiar; Programa de Postítulo de Medicina Familiar. (2008b). Informe de autoevaluación de los programas de medicina familiar.
Rojas P, Bittner M \& Rivera S. (2011). Protocolo: Evaluación del programa de formación médica UC de pregrado; caracterización y oportunidad de adquisición formal de competencias en el área psicosocial familiar orientadas a una atención clínica diferenciadora. FONDEDOC, Pontificia Universidad Católica de Chile.

Stewart M, Brown JB, Boon H, Galajda J, Meredith L \& Sangster M. (1999). Evidence on patient-doctor communication. Cancer prevention \& control : CPC = Prevention \& controle en cancerologie: PCC 3, 25-30.

Stewart M, Brown JB, Donner A, McWhinney IR, Oates J, Weston WW \& Jordan J. (2000). The impact of patient-centered care on outcomes. The Journal of family practice 49, 796-804.

Tannenbaum T, Konkin J, E. P, Saucier D, L. S, A. W, Kerr J \& Organek A. (2009). CanMEDS-Family Medicine, Working Group on Curriculum Review. College of Family Physicians of Canada. 